\title{
Corrigendum to: Module Arens Regularity for Semigroup Algebras
}

\author{
Reza Rezavand • Massoud Amini • \\ Mohammad Hossein Sattari • \\ Davood Ebrahimi Bagha
}

Received: 15 February 2009 / Accepted: 4 May 2009 / Published online: 11 June 2009

(C) Springer Science+Business Media, LLC 2009

\section{Erratum to: Semigroup Forum (2008) 77: 300-305 \\ DOI 10.1007/s00233-008-9075-3}

The last equivalence of the display in the proof of Theorem 2.2 (page 301) is not correct (and $b \in \mathcal{A}$ is missing inside the brackets of the fourth line of this display). As noted at the beginning of this section, $\mathcal{A}^{\prime}$ has a natural $\mathcal{O}$-module structure defined by

$$
\alpha . \lambda(a)=\lambda(a . \alpha), \quad \lambda . \alpha(a)=\lambda(\alpha . a) \quad\left(\alpha \in \mathcal{O}, a \in \mathcal{A}, \lambda \in \mathcal{A}^{\prime}\right) .
$$

We need to modify Definition 2.1 as follows.

Definition 2.1 $\mathcal{A}$ is called module Arens regular (as an $\mathcal{O}$-module) if the operator $\mathcal{R}_{\lambda}: \mathcal{A} \rightarrow \mathcal{A}^{\prime} ; a \mapsto a . \lambda$ is weakly compact for any $\lambda \in \mathcal{A}^{\prime}$ satisfying $\lambda(\alpha . a b)=$

Communicated by Jerome A. Goldstein.

The online version of the original article can be found under doi:10.1007/s00233-008-9075-3.

R. Rezavand · M. Amini ( $₫)$

Department of Mathematics, Tarbiat Modares University, Tehran 14115-175, Iran

e-mail: mamini@modares.ac.ir

R. Rezavand

e-mail: rezavand@modares.ac.ir

M.H. Sattari

Faculty of Basic Sciences, Azarbaijan University of Tarbiat Moallem, Tabriz, Iran e-mail: sattari@azaruniv.edu

D.E. Bagha

Faculty of Mathematical Sciences, Shahid Beheshti University, Evin, Tehran, Iran e-mail: d-ebrahimi@cc.sbu.ac.ir 
$\lambda(a b . \alpha), \lambda(c(\alpha . a b))=\lambda(c(a b . \alpha)), \lambda((\alpha . a b) d)=\lambda((a b . \alpha) d)$, and $\lambda(c(\alpha . a b) d)=$ $\lambda(c(a b . \alpha) d)$, for each $\alpha \in \mathcal{O}$ and $a, b, c, d \in \mathcal{A}$.

The conditions imposed on $\lambda$ in the above definition are equivalent to the assumption that the operators $\mathcal{R}_{\lambda}, \mathcal{R}_{\lambda . c}, \mathcal{R}_{d . \lambda}$, and $\mathcal{R}_{d . \lambda . c}$ are right $\mathcal{O}$-module homomorphisms, for each $a, b, c, d \in \mathcal{A}$. Now it is easy to show that $\lambda \in \mathcal{A}^{\prime}$ satisfies the above conditions if and only if $\lambda \in J^{\perp}$. This fixes the proof of Theorem 2.2. The rest of the paper is now correct. 